\title{
Estresse Hídrico e Salino na Germinação de Sementes de Anadenanthera colubrina (Veloso) Brenan
}

\section{Water and Salt Stress in the Germination of Anadenanthera colubrina (Veloso) Brenan Seeds}

\author{
Suelen Santos Rego ${ }^{1 *}$, Marcia Miriam Ferreira ${ }^{1}$, Antonio Carlos Nogueira ${ }^{1}$, Fernando \\ Grossi $^{1}$, Regiane Kock de Sousa ${ }^{1}$, Gilvano Ebling Brondani ${ }^{2}$, Marla Alessandra Araujo ${ }^{3}$ e \\ André Luís Lopes da Silva ${ }^{4}$
}

${ }^{1}$ Departamento de Ciências Florestais, Universidade Federal do Paraná - UFPR; 80.210-170, Curitiba - PR Brasil. ${ }^{2}$ Departamento de Ciências Florestais, Escola Superior de Agricultura "Luiz de Queiroz"/Universidade de São Paulo - ESALQ/USP; 13.418-900; Piracicaba - SP - Brasil. ${ }^{3}$ Departamento de Ciência do Solo, Universidade Federal de Lavras, Lavras, Minas Gerais, Câmpus Universitário, Caixa Postal 3037 CEP 37200-000 Lavras - MG, Brasil. ${ }^{4}$ Departamento de Engenharia de Bioprocessos e Biotecnologia, Universidade Federal do Paraná - UFPR, 81.531-970; Curitiba - PR - Brasil.

\begin{abstract}
The objective of this work was to verify levels of tolerance in Anadenanthera colubrina seeds to water and salt stress simulated for polyethyleneglycol 6000 (PEG 6000), manitol and $\mathrm{KCl}$. The seeds was collected from nine trees and stored in cold chamber at $5^{\circ} \mathrm{C}$. For the evaluation of the germination under it water stress was used PEG 6000 solutions and manitol, and under salt stress solutions of $\mathrm{KCl}$ in different osmotic potentials: $0.0 \mathrm{Mpa}$ (control), -0.6 $\mathrm{MPa},-0.8 \mathrm{MPa},-1.0 \mathrm{MPa},-1.2 \mathrm{MPa}$ and -1.4 Mpa. The seeds were made use in gerbox and placed in germination chambers, under constant light to the temperature of $25^{\circ} \mathrm{C}$. The percentage and the germination speed index (GSI) were evaluated. The seeds of Anadenanthera colubrina showed moderate tolerance to the water stress simulated by PEG 6000. The percentage of germination was affected from -1.0 MPa, and the GSI from -0.6 Mpa. Manitol did not reveal efficient in the simulation of water stress, therefore it did not reduce the percentage of germination. Under it salt stress, simulated for $\mathrm{KCl}$, the germination percentage was affected from the potential -1.2 MPa, characterizing a high limit of tolerance to this salt.
\end{abstract}

Key-words: Angico-branco, osmotic potential, salinity, polyethyleneglycol, manitol, $\mathrm{KCl}$

\section{INTRODUÇÃO}

Anadenanthera colubrina (Veloso) Brenan, pertencente à família Mimosaceae, é encontrada naturalmente na Floresta Estacional Semidecidual e menos frequente na Floresta Ombrófila Mista, Estacional Decidual, Pantanal Mato-Grossense e no Cerradão, onde é rara (Carvalho, 2003; Monteiro et al., 2006; Lucena et al., 2007; Albuquerque et al., 2011).

Ocorre desde áreas úmidas, em solos de boa disponibilidade hídrica, a secas como no Cerrado. Espécie pioneira a secundária inicial, heliófita, pode ser encontrada com maior frequência em florestas secundárias e ambientes alterados
(Justiniano e Fredericksen, 1998; Albuquerque et al., 2011). O angico-branco é uma planta com potencial apícola e medicinal, sendo que sua madeira pode ser utilizada na construção civil e naval, bem como para lenha e carvão. A árvore pode ser usada na arborização de estradas, parques e ruas. Esta espécie também pode ser recomendada para recuperação de áreas degradadas e para reposição de mata ciliar (Carvalho, 2003; Monteiro et al., 2006).

A uniformidade, velocidade e a porcentagem de emergência das plantas em campo apresentam significativos reflexos sobre a produção final. Fatores como temperaturas extremas, salinidade,

Author for correspondence: suelen_srego@yahoo.com.br

J. Biotec. Biodivers. v. 2, N.4: pp. 37-42, Nov. 2011 
excesso ou deficiência hídrica, crostas do solo e presença de insetos e patógenos podem afetar de forma adversa o estabelecimento das plantas no campo (Villela et al., 1991; Khan et al., 2001).

A primeira condição para a germinação de uma semente viável e não dormente é a disponibilidade de água para sua reidratação. Da absorção de água resulta a reidratação dos tecidos com a consequente intensificação da respiração e de todas as outras atividades metabólicas, que culminam com o fornecimento de energia $\mathrm{e}$ nutrientes necessários para a retomada de crescimento do eixo embrionário. Quanto maior a quantidade de água disponível para as sementes mais rápida será a absorção, sendo que a germinação não ocorre sob potenciais de água inferiores a determinado ponto crítico, que pode variar de acordo com a espécie (Bewley e Black, 1994).

O estresse hídrico em condições naturais pode atuar de forma positiva no estabelecimento das espécies, pois provoca um atraso considerável no tempo de germinação das sementes. Dessa forma, a germinação é distribuída no tempo e no espaço, aumentando a probabilidade das plântulas encontrarem condições ambientais adequadas ao estabelecimento e desenvolvimento (Bewley e Black, 1994).

O período de germinação e estabelecimento das plântulas arbóreas é importante para a sobrevivência das espécies florestais, principalmente nos locais onde a disponibilidade de água está limitada durante um período do ano. A capacidade das sementes de algumas espécies em germinar sob condições de estresse hídrico confere vantagens ecológicas em relação a outras que são sensíveis à seca (Rosa et al., 2005).

Em solos salinos, os sais afetam as plantas mediante a retenção osmótica de água e o efeito iônico específico sobre o protoplasma. As soluções salinas retêm água e, deste modo, reduzem o potencial hídrico, tornando-a cada vez menos acessível às plantas (Nasr et al., 2011). Esse efeito de solutos dissolvidos é similar a um déficit hídrico do solo e, a maioria das plantas responde à níveis excessivos de salinidade no solo da mesma forma para o déficit hídrico. Além disso, o excesso de sal no solo também pode causar efeitos de toxidez. Concentrações altas de sais totais nas células podem inativar as enzimas e inibir a síntese proteica (Taiz e Zeiger, 2004).

Algumas plantas possuem a habilidade de eliminar o excesso de sais, sem prejudicar seriamente a função vital. Estas tornam-se capazes de tolerar, em determinado grau de especificidade, dependendo da espécie e das proporções iônicas mudadas, os efeitos tóxicos e osmóticos associados ao estresse salino. A tolerância à salinidade pelas plantas torna-se importante na utilização de solos salinos para a agricultura e silvicultura (Bewley e Black, 1994).

Devido à carência de informações sobre a autoecologia das espécies tropicais nativas, existem dificuldades para adoção de práticas conservacionistas ou de recuperação de áreas degradadas (Carpi et al., 1996). Desse modo tornam-se fundamentais estudos sobre a germinação das sementes visando à utilização e exploração de forma racional das espécies nativas (Perez et al., 2001).

O objetivo deste trabalho foi verificar os níveis de tolerância das sementes de angico-branco ao estresse hídrico e salino simulados por PEG 6000, manitol e $\mathrm{KCl}$.

\section{MATERIAL E MÉTODOS}

As sementes de angico-branco foram coletadas de nove matrizes no município de Colombo - PR em julho de 2005 e armazenadas em câmara fria a 5 ${ }^{\circ} \mathrm{C}$. Foram amostradas 100 sementes para a determinação do peso de mil sementes. $\mathrm{O}$ teor de umidade foi obtido pelo método de estufa a $105^{\circ} \mathrm{C}$ $\left( \pm 3{ }^{\circ} \mathrm{C}\right)$ por 24 horas, por meio de três amostras de 50 sementes (Brasil, 1992).

Para a avaliação da germinação sob estresse hídrico foram utilizadas soluções de polietilenoglicol 6000 (PEG 6000) e manitol, e salino soluções de $\mathrm{KCl}$ sob diferentes potenciais osmóticos: 0,0 Mpa (testemunha), -0,6 MPa, - 0,8 $\mathrm{MPa},-1,0 \mathrm{MPa},-1,2 \mathrm{MPa}$ e -1,4 Mpa. As soluções de PEG 6000 foram preparadas de acordo com Vilella et al. (1991), e as de manitol e KCl preparadas a partir da fórmula de Van't Hoff, citada por Salysbury e Ross (1992):

$$
\Psi \text { os }=- \text { C i R T }
$$

Onde: $\Psi$ os $=$ potencial osmótico $($ atm),$\quad \mathrm{C}=$ concentração $\left(\right.$ mol. $\left.\mathrm{L}^{-1}\right), \mathrm{i}=$ coeficiente isotônico, $\mathrm{R}=$ constante geral dos gases perfeitos $\left(0,082 \mathrm{~atm} \cdot \mathrm{mol}^{-1}{ }^{\circ} \mathrm{K}^{-}\right.$ ${ }^{1}$ ) e $\mathrm{T}=$ temperatura $\left({ }^{\circ} \mathrm{K}\right)$.

As sementes foram dispostas em caixas plásticas do tipo gerbox previamente desinfestadas com solução hidroalcoólica a 70\% (v/v), contendo duas folhas de papel toalha esterilizadas e $10 \mathrm{ml} \mathrm{da}$ 
solução a ser testada. Os gerbox foram vedados com parafilme e dispostos em câmaras de germinação Biomatic, sob luz constante à temperatura de $25{ }^{\circ} \mathrm{C}$. A cada dois dias, as sementes foram transferidas de gerbox contendo novas soluções de polietilenoglicol 6000, manitol e $\mathrm{KCl}$ nas mesmas concentrações testadas, a fim de expor as sementes a níveis constantes dos potenciais osmóticos.

A contagem de sementes germinadas foi realizada diariamente durante 15 dias considerando-se como critério de germinação a emissão da radícula com no mínimo 2,0 mm (Rosa et al., 2005). Foram avaliados a porcentagem de germinação e o índice de velocidade de germinação (IVG), obtido pela fórmula proposta por Maguire (1962):

$$
\mathrm{IVG}=\frac{\sum \mathrm{ni}}{\mathrm{i}}
$$

Onde: $\mathrm{IVG}=$ índice de velocidade de germinação, $\mathrm{ni}=$ número de sementes germinadas no dia, $\mathrm{i}=$ número de dias após a instalação do teste.

O delineamento experimental adotado foi inteiramente casualizado, em esquema bifatorial ( 3 $\mathrm{x}$ 6), sendo os fatores constituídos por três soluções e por seis potenciais osmóticos, com seis repetições de 25 sementes para cada tratamento.
Os dados referentes a porcentagem de germinação e ao índice de velocidade de germinação foram submetidos ao teste de Bartlett para a verificação da homogeneidade da variância entre os tratamentos $(P<0,05)$. Em seguida, realizou-se análise de variância (ANOVA, $P<0,05$ e $P<0,01$ ). De acordo com a significância, os dados foram submetidos à análise de regressão polinomial $(P<0,05)$.

\section{RESULTADOS E DISCUSSÃO}

Para as análises físicas das sementes obteve-se o peso de mil sementes de 50,5 g $(\mathrm{CV}=5,15 \%)$, o que correspondeu a estimativa de 19.797 sementes $\mathrm{kg}^{-1}$ com $12,5 \%$ de umidade.

$\mathrm{O}$ teste de Bartlett revelou homogeneidade de variância para a característica da porcentagem de germinação (PG). Porém, os valores do índice de velocidade de germinação (IVG) não apresentaram esse comportamento, sendo necessária a transformação dos dados previamente a análise de variância (Tabela 1).

A ANOVA revelou significância a $1 \%$ de probabilidade de erro para todas as características analisadas, sendo constatada interação entre a solução testada com a variação do potencial osmótico (Tabela 1).

Tabela 1. Resumo da análise de variância (ANOVA) para as características da porcentagem de germinação (PG) e índice de velocidade de germinação (IVG) de sementes de Anadenanthera colubrina, em função dos tratamentos testados.

\begin{tabular}{lccc}
\multicolumn{1}{c}{ Causas da Variação } & \multirow{2}{*}{ GL } & \multicolumn{2}{c}{ Quadrados Médios } \\
\hline Solução $($ Sol) & 2 & $18.528,59^{* *}$ & $\mathrm{IVG}^{(1)}$ \\
Potencial Osmótico (Pos) & 5 & $4.289,30^{* *}$ & $0,2318^{* *}$ \\
Sol * Pos & 10 & $1.542,99^{* *}$ & $0,0858^{* *}$ \\
Resíduo & 90 & 75,73 & $0,0108^{* *}$ \\
Média & - & $57,85 \%$ & 0,0003 \\
$\mathrm{CV}_{\text {exp. }}(\%)$ & - & 15,04 & 7,66 \\
\hline${ }^{* *}$ valor de F significativo ao nível de $1 \%$ de probabilidade de erro. ${ }^{(1)}$ dados transformados por $\sqrt{\frac{(\mathrm{p}+1)}{100}}$ pelo teste de
\end{tabular}

Bartlett, ao nível de $5 \%$ de probabilidade de erro. $\mathrm{GL}=$ graus de liberdade, $\mathrm{CV}_{\text {exp. }}=$ coeficiente de variação experimental, $\mathrm{Sol}=$ solução, $\mathrm{Pos}=$ potencial osmótico.

A maior média referente a porcentagem de germinação foi observada para a testemunha $(0$ $\mathrm{MPa}$ ) que apresentou $75,3 \%$ de germinação. Nos tratamentos com PEG 6000 a porcentagem de germinação foi reduzida a partir do potencial de $0,6 \mathrm{Mpa}(62,7 \%)$, sendo que a partir do potencial de -1,0 Mpa a porcentagem de germinação reduziu drasticamente $(14 \%)$ chegando a zero nos potenciais de $-1,2$ e $-1,4 \mathrm{Mpa}$, verificando um limite de tolerância para a germinação entre $-1,0$ e -1,2 Mpa (Figura 1). 


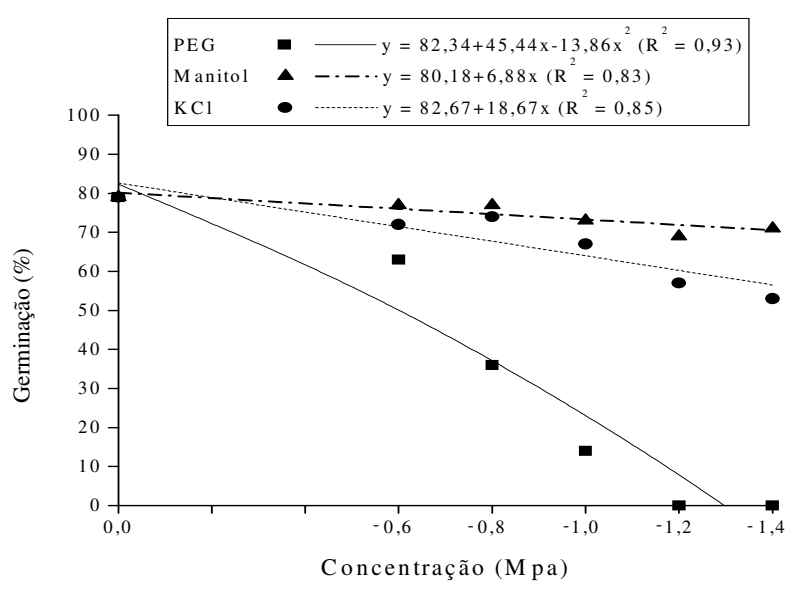

Figura 1 - Porcentagem de germinação de sementes de Anadenanthera colubrina submetidas a soluções de PEG 6000, manitol e $\mathrm{KCl}$ em diferentes potenciais osmóticos.

Em relação ao índice de velocidade de germinação, observou-se redução em seus valores a partir do potencial de $-0,6 \mathrm{Mpa}$, o que correspondeu a estimativa de 4,2 para o tratamento com PEG (Figura 2).

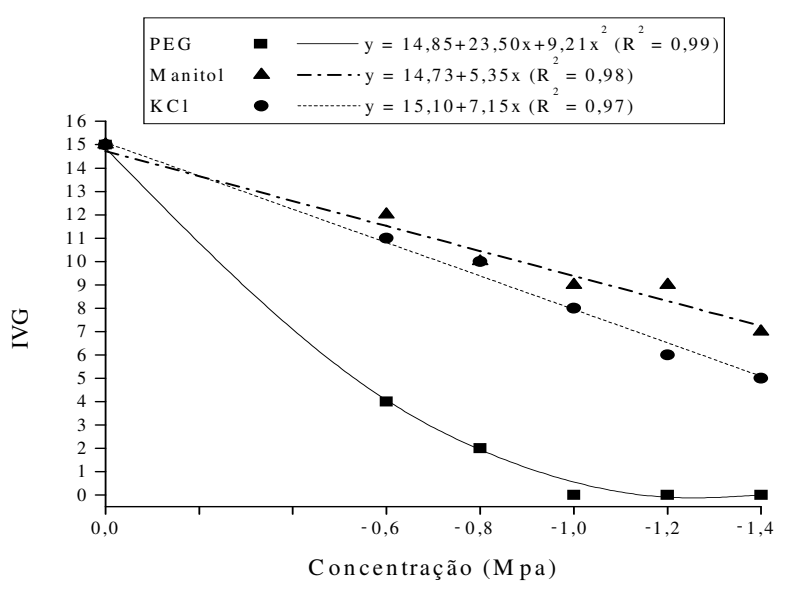

Figura 2 - Índice de velocidade de germinação de sementes de Anadenanthera colubrina submetidas a soluções de PEG 6000, manitol e $\mathrm{KCl}$ em diferentes potenciais osmóticos.

As sementes de angico-branco apresentam moderada tolerância ao estresse hídrico simulado por PEG 6000, sendo que as respostas podem variar muito de acordo com o material genético avaliado (Khan et al., 2001; Nasr et al., 2011).

Resultados semelhantes foram constatados por Silva et al. (2005) que verificaram limites reduzidos de tolerância ao estresse hídrico para as sementes de Cnidosculus juercifolius (faveleira). Estes autores condicionaram osmoticamente as sementes aos potenciais de $-0,1$ a $-1,3 \mathrm{MPa}$, sendo a porcentagem de germinação afetada a partir de $0,5 \mathrm{MPa}$ e a velocidade de germinação reduzida a partir de -0,3 Mpa, sendo que os valores compreendidos entre $-0,9$ e -1,3 MPa a germinação foi completamente inibida.

Fanti et al. (2004) testando potenciais de 0 a $-0,7$ Mpa em sementes de Chorisia speciosa (paineira) verificaram que a partir do potencial de $-0,5 \mathrm{Mpa}$ verificaram decréscimo significativo da porcentagem de germinação, sendo que a partir do potencial $-0,7 \mathrm{Mpa}$ a germinação foi nula.

Para a espécie Ateleia glazioviana (timbó), Rosa et al. (2005) estudaram potenciais osmóticos compreendidos entre $-0,2$ a $-1,0 \mathrm{MPa}$ induzido por PEG 6000 e, verificaram que a partir de $-0,4 \mathrm{MPa}$ existiu decréscimo para a germinação, sendo mais acentuando para os potenciais de - 0,6 e $-0,8 \mathrm{Mpa}$ e nula para o potencial $-1,0 \mathrm{MPa}$. Na mesma linha de trabalho, Perez et al. (2001) obtiveram limites ainda maiores de tolerância para Peltophorum dubium (canafístula) ao testarem potenciais de 0 a -2,0 MPa, sendo que os limites de tolerância para a germinação situaram-se entre -1,4 e -1,6 MPa, o que serviu de base para classificar a espécie como resistente ao estresse hídrico simulado por PEG 6000.

Várias espécies resistem a variações do potencial hídrico, sendo que as mais resistentes possuem a vantagem ecológica de estabelecer suas plântulas em áreas onde as mais sensíveis não germinariam (Bewley e Black, 1994). Essa característica tornase importante para fins de recomendações para o plantio de espécies capazes de suportar diferentes condições de potenciais osmóticos em diversas situações ecológicas, principalmente ao considerar os solos salinos e áreas com baixa disponibilidade hídrica.

As sementes de angico-branco expressaram níveis maiores de tolerância ao estresse hídrico simulado por manitol em relação ao simulado por PEG 6000 , pois para os tratamentos com manitol não se verificou diferença na porcentagem de germinação em nenhum dos potenciais avaliados (Figura 1). No entanto, houve redução no índice de velocidade de germinação a partir do potencial de $-0,6 \mathrm{Mpa}$ (Figura 2). Desta forma verifica-se que o manitol não se mostrou eficiente na simulação de estresse hídrico.

Nassif e Perez (1997) e Fanti et al. (2004) também 
verificaram maior tolerância das sementes de Pterogyne nitens (amendoim-do-campo) e Chorisia speciosa (paineira), respectivamente, ao estresse hídrico simulado por manitol em relação ao simulado por PEG 6000. De acordo com Fanti et al. (2004) esse efeito deve-se a permeabilidade diferencial do tegumento das sementes aos solutos de baixo peso molecular, que teria favorecido a entrada de manitol nas sementes, reduzindo o verdadeiro efeito de seca. Para Villela et al. (1991) consideraram o polietilenoglicol 6000 como o soluto mais indicado para simular o controle da hidratação, pois o mesmo não penetra nos tecidos, fator esse que pode ter influenciado as respostas em Anadenanthera colubrina.

Sob estresse salino, as sementes de angico-branco apresentaram limite elevado de tolerância. Nos tratamentos com $\mathrm{KCl}$ resultados satisfatórios de germinação foram encontrados até o potencial de 1,0 Mpa (66,6\%), sendo que a porcentagem de germinação foi reduzida somente a partir do potencial de -1,2 Mpa (57,3\%) (Figura 1). Para o índice de velocidade de germinação houve um decréscimo a partir do potencial de -06 Mpa (Figura 2).

Resultados semelhantes foram encontrados por Nassif e Perez (1997) que classificaram as sementes de Pterogyne nitens (amendoim-docampo) resistentes ao estresse salino simulado por $\mathrm{KCl}$, ao verificarem reduções significativas da germinação e da velocidade de germinação a partir de -1,2 Mpa e -0,6 Mpa, respectivamente. Fanti et al. (2004) obtiveram níveis menores de tolerância ao $\mathrm{KCl}$ para sementes de Chorisia speciosa (paineira); sendo que reduções significativas da germinação ocorreram em -0,87 Mpa. Segundo Bewley e Black (1994) um grau moderado de resistência ao sal é útil na tentativa de utilização de solos salinos em regiões secas, visto que $6 \%$ dos continentes terrestres constituem-se de solos salinos.

O conhecimento das espécies capazes de suportar tais condições pode auxiliar na adequada recomendação para o plantio em situações de estresse salino, principalmente ao considerar as espécies nativas, onde há escassez de informações, existindo a necessidade de maior número de estudos direcionados a essa linha de pesquisa.

\section{CONCLUSÕES}

Os resultados demonstraram que as sementes de angico-branco possuem moderada tolerância ao estresse hídrico simulado por PEG 6000.
O manitol não foi eficiente na simulação de estresse hídrico.

Sob estresse salino, simulado por $\mathrm{KCl}$, as sementes de angico-branco obtiveram limite elevado de tolerância.

\section{AGRADECIMENTOS}

À Embrapa-Florestas e a CAPES pelo apoio concedido.

\section{RESUMO}

O objetivo deste trabalho foi avaliar os níveis de tolerância das sementes de angico-branco ao estresse hídrico e salino simulados por polietilenoglicol 6000 (PEG 6000), manitol e KCl. As sementes foram coletadas de nove matrizes no município de Colombo PR e armazenadas em câmara fria a $5{ }^{\circ} \mathrm{C}$. Para a avaliação da germinação sob estresse hídrico foram utilizadas soluções de PEG 6000 e manitol, e sob estresse salino, soluções de $\mathrm{KCl}$ nos seguintes potenciais osmóticos: 0,0 Mpa (testemunha), -0,6 MPa, $-0,8 \mathrm{MPa},-1,0 \mathrm{MPa},-1,2 \mathrm{MPa}$ e -1,4 Mpa. As sementes foram dispostas em gerbox e acondicionadas em câmaras de germinação, sob luz constante à temperatura de $25^{\circ} \mathrm{C}$. Foram avaliados a porcentagem e o índice de velocidade de germinação. As sementes de angicobranco apresentaram moderada tolerância ao estresse hídrico simulado por PEG 6000. A porcentagem de germinação foi afetada drasticamente a partir de $-1,0$ MPa e, a velocidade de germinação a partir de -0,6 Mpa. O manitol não foi eficiente na simulação de estresse hídrico, pois não reduziu a porcentagem de germinação em nenhum dos potenciais testados. Sob estresse salino, simulado por $\mathrm{KCl}$, a porcentagem de germinação foi afetada a partir do potencial -1,2 MPa, caracterizando limite elevado de tolerância a este sal.

Palavras-chave: angico-branco, potencial osmótico, salinidade, polietilenoglicol, manitol, $\mathrm{KCl}$

\section{REFERÊNCIAS}

Albuquerque, U. P.; Soldati, G. T.; Sieber, S. S.; Medeiros, P. M.; Sá, J. C.; Souza, L. C. (2011), Rapid ethnobotanical diagnosis of the Fulni-ô Indigenous lands (NE Brazil): floristic survey and local conservation priorities for medicinal plants. Environment, Development and Sustainability, 13, 277-292.

Bewley, J. D. e Black, M. (1994), Seeds: physiology of development and germination. New York: Plenum Press, 445p.

Brasil (1992), Ministério da Agricultura e Reforma Agrária. Regras para análise de sementes. Brasília: SNDA/DNDV/CLAV, 365p. 
Carpi, S. M. F.; Barbedo, C. J.; Marcos Filho, J. (1996), Condicionamento osmótico de sementes de Cedrela fissilis Vell. Revista Brasileira de Sementes, 18, 271-275.

Carvalho, P. E. R. (2003), Espécies arbóreas brasileiras. Colombo: Embrapa Florestas. 1, $1039 \mathrm{p}$.

Fanti, S. C. e Perez, S. C. J. G. A. (2004), Processo germinativo de sementes de paineira sob estresses hídrico e salino. Pesquisa Agropecuária Brasileira, 39, 903-909.

Justiniano, M. J. e Fredericksen, T. S. (1998), Ecologia y silvicultura de especies menos conocidas: curupaú - Anadenanthera colubrina (Vell. Conc.) Benth. Mimosoideae. Santa Cruz: BOLFOR, 31p.

Khan, M. A.; Gul, B.; Weber, D. J. (2001), Influence of salinity and temperature on the germination of Kochia scoparia. Wetlands Ecology and Management, 9, 483-489.

Lucena, R. F. P.; Albuquerque, U. P.; Monteiro, J. M.; Almeida, C. F. C. B. R.; Florentino, A.T.N.; Ferraz, J.S.F. (2007), Useful plants of the SemiArid Northeastern Region of Brazil - a look at their conservation and sustainable use. Environmental Monitoring and Assessment, 125, 281-290.

Maguire, J. D. (1962), Speed of germination aid in selection and evaluation for seedling emergence and vigor. Crop Science, 2, 176-177.

Monteiro, M. J.; Almeida, C. F. C. B. R.; Albuquerque, U. P.; Lucena, R. F. P.; Florentino, A. T. N.; Oliveira, R. L. C. (2006), Use and traditional management of Anadenanthera colubrina (Vell.) Brenan in the semi-arid region of northeastern Brazil. Journal of Ethnobiology and Ethnomedicine, 2, 1-7.
Nasr, S. M. H.; Parsakhoo, A.; Naghavi, H.; Koohi, S. K. S. (2011), Effect of salt stress on germination and seedling growth of Prosopis juliflora (Sw.). New Forests, 42, 9265-9269.

Nassif, S. M. L. e Perez, S. C. J. G. A. (1997), Germinação de sementes de amendoim-do-campo (Pterogyne nitens Tul. - FabaceaeCaesalpinoideae) submetidas a diferentes condições de estresse hídrico e salino. Revista Brasileira de Sementes, 19, 143-150.

Perez, S. C. J. G. A.; Fanti, S. C.; Casali, C. A. (2001), Influência da luz na germinação de sementes de canafístula submetidas ao estresse hídrico. Bragantia, 60, 155-156.

Rosa, L. S.; Felippi, M.; Nogueira, A. C.; Grossi, F. (2005), Avaliação da germinação sob diferentes potenciais osmóticos e caracterização morfológica da semente e plântula de Ateleia glazioviana Bail (Timbó). Revista Cerne, 11, 306-314.

Salysbury, F. B. e Ross, C. W. (1992), Plant physiology. $4^{\text {th }}$ ed. Belmont: Wadsworth Publishing Company, 682p.

Silva, L. M. M.; Aguiar, I. B.; Morais, D. L.; Viégas, R. A. (2005), Estresse hídrico e condicionamento osmótico na qualidade fisiológica de sementes de faveleira (Cnidoscolus juercifolius). Revista Brasileira de Engenharia Agrícola, 9, 66-72.

Taiz, L. e Zeiger, E. (2004), Fisiologia vegetal. 3 ed. Porto Alegre: Artmed, 719p.

Villela, F. A.; Doni Filho, L.; Siqueira, E. L. (1991), Tabela do potencial osmótico em função da concentração de polietileno glicol 6000 e da temperatura. Pesquisa Agropecuária Brasileira, 26, 1957-1968. 